\title{
Haben Patient*innen die moralische Pflicht, ihre klinischen Daten für Forschung bereitzustellen? Eine kritische Prüfung möglicher Gründe
}

\author{
Martin Jungkunz $\mathbb{D} \cdot$ Anja Köngeter $(\mathbb{D} \cdot$ Katja Mehlis $(\mathbb{D} \cdot$ Markus Spitz • \\ Eva C. Winkler $(\mathbb{D} \cdot$ Christoph Schickhardt $(\mathbb{D}$
}

Eingegangen: 6. August 2021 / Angenommen: 4. Januar 2022 / Online publiziert: 25. Februar 2022

(C) Der/die Autor(en) 2022

Zusammenfassung Die Sekundärnutzung klinischer Daten für Forschungs- und Lernaktivitäten hat das Potenzial, medizinisches Wissen und klinische Versorgung erheblich zu verbessern. Zur Realisierung dieses Potenzials bedarf es einer ethischen und rechtlichen Grundlage für die Datennutzung, vorzugsweise in Form der Einwilligung von Patient*innen. Damit stellt sich die grundsätzliche Frage: Haben Patient*innen eine moralische Pflicht, ihre klinischen Daten für Forschungs- und Lernaktivitäten zur Verfügung zu stellen?

Auf Basis eines ethischen Ansatzes, der als „sorgender Liberalismus“ bezeichnet werden kann, werden folgende Argumente zur Begründung einer Pflicht von Patient*innen zur Bereitstellung ihrer klinischen Daten für Forschungs- und Lernaktivitäten auf Plausibilität und moralisches Gewicht untersucht: die allgemeine Hilfspflicht; Solidarität; die Pflicht zu gemeinwohlförderlichem Handeln; das Trittbrettfahrerargument; transgenerationale Gerechtigkeit; das Prinzip des Zurückgebens; das Prinzip des Nicht-Schädigens; die Forschungsfreiheit und der Wert der Wissenschaft.

Die allgemeine Hilfspflicht und die Pflicht zu gemeinwohlförderlichem Handeln sind gewichtige Gründe für eine moralische Pflicht von Patient*innen zur Bereitstellung ihrer klinischen Daten für Forschungs- und Lernaktivitäten. Das Argument der

Dr. Martin Jungkunz ( $\bowtie) \cdot$ Dr. Christoph Schickhardt

Sektion für Translationale Medizinethik, Medizinische Onkologie, Deutsches

Krebsforschungszentrum (DKFZ), Nationales Centrum für Tumorerkrankungen (NCT), Im

Neuenheimer Feld 460, 69120 Heidelberg, Deutschland

E-Mail: martin.jungkunz@dkfz-heidelberg.de

Anja Köngeter, M.A. · Dr. Katja Mehlis · Prof. Dr. Dr. Eva C. Winkler

Sektion für Translationale Medizinethik, Medizinische Onkologie, Universitätsklinikum Heidelberg,

Nationales Centrum für Tumorerkrankungen (NCT), Im Neuenheimer Feld 460, 69120 Heidelberg,

Deutschland

RA Markus Spitz

Juristische Fakultät, Ruprecht Karls-Universität Heidelberg, Heidelberg, Deutschland 
transgenerationalen Gerechtigkeit und das Prinzip des Zurückgebens sind ethisch schwache Gründe für eine solche Pflicht, können jedoch eine motivationale Rolle spielen. Die anderen Gründe sind nicht geeignet, eine Pflicht zu begründen. Das Ergebnis ist in mehrfacher Hinsicht relevant: für Patient*innen, die um die Einwilligung in die Sekundärnutzung ihrer klinischen Daten gebeten werden; für die ethische Diskussion der Frage, ob und inwieweit Abstriche von der klassischen spezifischen Einwilligung unter bestimmten Bedingungen ethisch akzeptabel sind; für die rechtwissenschaftliche Diskussion der Bedingungen für eine juristisch verhältnismäßige Sekundärnutzung klinischer Daten für Forschungs- und Lernaktivitäten.

Schlüsselwörter Sekundärnutzung · Patientendaten · Moralische Pflicht · Datenteilen · Biomedizinische Forschung

\title{
Do patients have a moral duty to provide their clinical data for research? A critical examination of possible reasons
}

\begin{abstract}
Research question The secondary use of clinical data for research and learning activities has the potential to significantly improve medical knowledge and clinical care. To realize this potential, an ethical and legal basis for data use is needed, preferably in the form of patient consent. This raises the question: Do patients have a moral duty to provide their clinical data for research and learning activities?

Methods On the basis of an ethical approach that we call "caring liberalism," we evaluate plausibility and moral weight of the following arguments in favor of a duty on patients to provide their clinical data for research and learning activities: the general duty to help; solidarity; the duty to act in the public interest; the free-rider argument; transgenerational justice; the principle of giving back; the do-not-harm principle; freedom of research and the value of science.

Results The general duty to help and the duty to act in the public interest are strong reasons for a moral duty of patients to provide their clinical data for research and learning activities. Transgenerational justice and the principle of giving back are ethically weak reasons for such a duty but might be motivational factors. The other reasons are not appropriate to justify a duty. The results are relevant in several respects: for patients who are asked to consent to the secondary use of their clinical data; for the ethical discussion of the question whether and to what extent deviations from the classical specific consent are ethically justifiable under certain conditions; for the legal discussion of the conditions for a proportionate secondary use of clinical data for research and learning activities.
\end{abstract}

Keywords Secondary use $\cdot$ Patient data $\cdot$ Moral duty $\cdot$ Data sharing $\cdot$ Biomedical research 


\section{Einleitung}

Die Sekundärnutzung klinischer Daten in datensammelnden, nicht-interventionellen Forschungs- und Lernaktivitäten - auf Englisch ,secondary use of clinical data in data-gathering, non-interventional research or learning activities“ (Jungkunz et al. 2021), daher im Folgenden abkürzend als ,SeConts“ bezeichnet - birgt großes Potenzial. Es wird allgemein angenommen, dass SeConts einen wichtigen Beitrag zur Gewinnung neuer wissenschaftlicher Erkenntnisse in der Biomedizin und zur Verbesserung der klinischen Versorgung leisten kann. International existieren zahlreiche Initiativen zur Ermöglichung und Förderung von SeConts (Hulsen 2020). In Deutschland strebt die Medizininformatik-Initiative (MII) ${ }^{1}$ den Aufbau einer digitalen Infrastruktur zwischen allen Universitätsklinika an, um klinische Daten vernetzt nutzbar und somit die systematische Durchführung von SeConts möglich zu machen. Voraussetzung für die Realisierung der Potenziale von SeConts ist jedoch nicht allein die technische und organisatorische Infrastruktur. SeConts ist auch abhängig davon, dass die klinischen Daten der Patient*innen genutzt werden dürfen, z. B. auf Grundlage einer Einwilligung seitens der Patient*innen. Aus ethischer Sicht stellt sich die Frage, ob Patient*innen angesichts der Nutzenpotenziale von SeConts ihre klinischen Daten zur Verfügung stellen sollen. Die Fragestellung der vorliegenden Arbeit lautet: Welche Gründe für eine moralische Pflicht von Patient*innen, ihre Daten für SeConts bereitzustellen, kommen in Betracht und wie sind sie im Einzelnen ethisch zu bewerten?

Die Beantwortung dieser ethischen Frage ist in mehrfacher Hinsicht relevant und dringlich: erstens für Patient*innen, denen in Zukunft vermehrt die Frage gestellt werden wird, ob sie in die Bereitstellung ihrer Daten für SeConts einwilligen. Relevanz besteht zweitens für die Debatte darüber, ob und inwieweit Abstriche vom Ideal der klassischen (spezifischen) Form der informierten Einwilligung, z. B. im Falle einer breiten Einwilligung oder gar einer systematischen Nutzung ohne explizite Einwilligung und nur mit Widerspruchsmöglichkeit (Opt-Out) ethisch akzeptabel sind. Drittens ist die folgende ethische Analyse auch für die rechtwissenschaftliche und rechtsethische Diskussion der Frage relevant, welche Bedingungen für eine juristisch verhältnismäßige Weiterverarbeitung klinischer Daten für Forschungszwecke erfüllt werden müssen. ${ }^{2}$

Die Frage nach Gründen für eine moralische Pflicht von Patient*innen, klinische Daten für SeConts bereitzustellen, hat bisher keine große Aufmerksamkeit in der bioethischen Literatur erfahren. Die meisten Texte, die auf die Frage eingehen, streifen sie nur als einen Aspekt im Rahmen einer anderen übergeordneten Fragestellung. Porsdam Mann et al. (2016) gehen bei der Ausarbeitung eines ethischen Rahmenwerks für die Sekundärnutzung klinischer Daten davon aus, dass Patient*innen im Rahmen einer Rettungspflicht (duty to easy rescue) die Pflicht haben, ihre Daten für SeConts bereitzustellen. Ballantyne und Schaefer (2018) argumentieren mit Blick auf die Notwendigkeit einer informierten Einwilligung für SeConts mit Daten aus einem öffentlichen Gesundheitssystem, dass die Gesellschaft einen legitimen An-

\footnotetext{
1 https://www.medizininformatik-initiative.de/. Zugegriffen: 7. April 2021.

2 Vgl. hierzu Kielmansegg (2020).
} 
spruch auf die Datennutzung in SeConts hat. Faden et al. (2013) führen die Pflicht von Patient*innen, klinische Daten für SeConts bereitzustellen, als eine von insgesamt sieben Pflichten für die Stakeholder eines noch zu etablierenden zukünftigen Lernenden Gesundheitssystems an. Nur Cohen (2018) geht der Frage ausführlicher nach und sieht Patient*innen in der Pflicht, ihre klinischen Daten für SeConts bereitzustellen, da sie selbst von SeConts profitieren. Eine weitere Limitierung der Literatur besteht darin, dass die einzelnen Beiträge zur Debatte alle jeweils nur auf ein einzelnes Argument eingehen, um eine Pflicht von Patient*innen, ihre klinischen Daten für SeConts bereitzustellen, zu begründen, und dabei mögliche weitere Gründe unbeachtet lassen. Im Gegensatz dazu hat der vorliegende Artikel den Anspruch, ein breiteres Spektrum an potenziellen Gründen für eine Pflicht von Patient*innen, ihre klinischen Daten für SeConts bereitzustellen, in den Blick zu nehmen und im Rahmen eines systematischen Ansatzes jeden einzelnen Grund ethisch zu analysieren und zu bewerten. Hierzu greifen wir drei Arten von Gründen auf: (1) die soeben angesprochenen Gründe aus der bestehenden Debatte um die Pflicht, klinische Daten für SeConts bereitzustellen, (2) Gründe aus der bioethischen Diskussion um eine mögliche moralische Pflicht von Patient*innen, an interventioneller klinischer Forschung teilzunehmen und (3) weitere Gründe, die wir in die Diskussion einführen, da wir sie für ethisch plausibel halten.

Der Artikel gliedert sich wie folgt: Einführend werden wichtige Begrifflichkeiten geklärt, SeConts wird an einem konkreten Beispiel veranschaulicht und der ethischnormative Hintergrund wird erläutert. Daraufhin werden die Risiken und der potenzielle Nutzen von SeConts dargelegt. Im anschließenden Hauptteil werden mögliche Gründe für eine Pflicht von Patient*innen, ihre klinischen Daten für SeConts bereitzustellen, auf ihre Plausibilität hin überprüft. Im letzten Abschnitt findet sich als erstes Ergebnis ein Gesamtrückblick auf die einzelnen Gründe für eine mögliche Pflicht von Patient*innen, ihre klinischen Daten für SeConts bereitzustellen. Als zweites Ergebnis wird in einer Art Ausblick dafür argumentiert, dass es ausreichend Gründe für eine prima facie Pflicht gibt. Zuletzt wird die praktische Relevanz dieser Ergebnisse erläutert.

\section{Hintergrund}

Die Daten, um deren Bereitstellung es in dieser Arbeit geht, werden im Zuge der Diagnostik und Behandlung von Patient*innen innerhalb eines öffentlichen Gesundheitssystems erzeugt, wie es für viele europäische Länder typisch ist. Konkret bezieht sich die Fragestellung dieses Artikels auf die Daten gesetzlich versicherter Patient*innen im deutschen Gesundheitssystem. Die meisten Ausführungen des Artikels gelten jedoch auch für die Mitglieder privater Krankenversicherungen. Unter den synonym gebrauchten Begriffen ,bereitstellen“ und ,zur Verfügung stellen“ klinischer Daten wird im Folgenden sowohl das Erteilen einer Einwilligung in die Datennutzung vonseiten der Patient*innen verstanden (z. B. durch eine breite Einwilligung) als auch eher passiv das Nicht-Wahrnehmen der Widerspruchsmöglichkeit (Opt-Out) im Falle einer systematischen Durchführung von SeConts ohne explizite Einwilligung. 
Den Ablauf der Verwendung der Daten in SeConts kann man sich beispielhaft - angelehnt an die MII - folgendermaßen vorstellen: Klinische Daten, die im Zuge der Diagnostik und Behandlung im klinischen Informationssystem der behandelnden Klinik hinterlegt werden, werden für Forschungszwecke zusätzlich im sogenannten Datenintegrationszentrum (DIZ) des jeweiligen Klinikstandorts gespeichert. Externe Forscher*innen können nun beim DIZ einen Antrag auf Datennutzung stellen, welcher, nach Prüfung und Bewilligung durch ein Datennutzungs- und Zugangskomitee, in einem vertraglich festgelegten Rahmen genehmigt werden kann.

Die hier vorgebrachten ethischen Analysen erfolgen aus einer ethisch-normativen Perspektive, die man als ,sorgenden Liberalismus“ bezeichnen kann (Fleischer et al. 2016) und die den Versuch darstellt, Rawls' sogenannten egalitären Liberalismus (Rawls 1979) für das Feld der anwendungsorientierten Forschungs- und Medizinethik weiterzuentwickeln. Dabei wird der Fürsorge, die, sei es als Prinzip oder als Tugend, für die Ethik in Medizin und Forschung von großer Bedeutung ist, angemessen und in einem liberalen, nicht-paternalistischen Sinne Rechnung getragen. Auf grundlegender Ebene sieht der sorgende Liberalismus in Anknüpfung an die klassische liberale Denklinie die Menschen als Träger von gleichen Grundfreiheiten, zu denen neben klassischen Freiheitsrechten wie der Meinungs-, Religions- und Gewissensfreiheit auch der Schutz der Privatsphäre und die informationelle Selbstbestimmung gehören. Die Einschränkung individueller Grundfreiheiten bedarf stets einer Rechtfertigung und ist nur dann legitim, wenn dadurch die Grundfreiheiten anderer Individuen oder Grundgüter (wie Bildung oder Gesundheit) ${ }^{3}$ wesentlich geschützt oder gefördert werden. Im Sinne der Fürsorge hat das Individuum die moralische Pflicht, andere Individuen dabei zu unterstützen, die nötigen Mittel und Voraussetzungen zu erlangen, um Grundfreiheiten im Sinne eines selbstbestimmten Lebens auch wirklich auszuüben. Auf gesellschaftlich-politischer Ebene hat das Individuum die Pflicht zur Unterstützung der demokratisch-rechtsstaatlichen Institutionen - die ihrerseits u.a. die Freiheiten der Bürger*innen schützen und den Einzelnen dabei unterstützen, ein selbstbestimmtes Leben zu führen.

\section{Risiken und Nutzen von SeConts}

Die Risiken und der potenzielle Nutzen von SeConts sind mehr oder weniger für die ethische Analyse aller Argumente bezüglich einer möglichen Pflicht von Patient*innen, ihre Daten für SeConts bereitzustellen, relevant, weshalb sie an dieser Stelle gezielt vorab erläutert werden. Hierbei beschränken wir uns auf Risiken für Patient*innen, da es im Artikel um sie bzw. ihre mögliche Pflicht geht. ${ }^{4}$

Elementar für die Bewertung der Risiken für Patient*innen ist die Art der in SeConts verwendeten Daten. Vor der Herausgabe der Daten an SeConts werden die Daten der Patient*innen de-identifiziert, d.h. es werden alle direkt identifizierenden Attribute wie Name, Adresse oder Geburtsdatum aus den Daten gelöscht (und

\footnotetext{
3 Zur genaueren Bestimmung von Grundgütern, vgl. Bohlken (2011).

${ }^{4}$ Mögliche Risiken für andere Beteiligte wie Ärzt*innen und Kliniken (vgl. Jungkunz et al. 2022) sind an dieser Stelle nicht relevant und werden daher auch nicht erläutert.
} 
ggf. durch Codes ersetzt). De-identifizierte Daten sind jedoch nicht notwendigerweise anonym. Zwar können einzelne Daten, wie z.B. Blutdruckwerte, durchaus anonym sein. Jedoch benötigt Forschung in aller Regel Datensätze, die Krankheitsgeschichten in Breite und Tiefe abbilden ${ }^{5}$ und aufgrund ihrer Feingranularität in der Regel nicht mehr als anonym gelten können. ${ }^{6}$ Für die Patient*innen kann also eine mögliche Verletzung der Vertraulichkeit ihrer Daten durch unerlaubte Re-Identifizierung nicht mit Sicherheit ausgeschlossen werden. Im Falle einer unbefugten Re-Identifizierung sind weitere negative Konsequenzen für Patient*innen denkbar, z.B. verschiedene Formen des Datenmissbrauchs, angefangen von personalisierter Werbung bis hin zu Diskriminierung, Identitätsdiebstahl und Erpressung. Als ein weiteres Risiko ist theoretisch auch ein negativer Effekt auf die Arzt-PatientenBeziehung denkbar, wenn Patient*innen aus Sorge um den Schutz ihrer Daten in SeConts das Vertrauen in die Privatheit der Kommunikation mit ihren Ärzt*innen verlieren. Auf eine solche Sorge können Patient*innen allerdings reagieren, indem sie der Datennutzung in SeConts widersprechen (durch Verweigerung der Einwilligung oder Opt-Out) - weshalb diesem Risiko hier nicht weiter nachgegangen wird. Die Eintrittswahrscheinlichkeit der anderen genannten, informationellen Risiken in SeConts lässt sich zurzeit nicht leicht einschätzen. Dennoch erscheint es aus folgenden Gründen plausibel, dass SeConts, zumindest in Deutschland, keine signifikante Erhöhung der abstrakt bestehenden Datenschutzrisiken darstellt, die bereits durch die Speicherung der Daten im Rahmen der klinischen Versorgung bestehen: (1) Bisher sind wenig Berichte über Datenpannen oder -diebstahl im Forschungskontext bekannt geworden. (2) Klinische Daten liegen bei SeConts - im Gegensatz zu elektronischen Dokumentationssystemen in Kliniken und Arztpraxen - in der Regel deidentifiziert vor. Eine Re-identifizierung von Patient*innen auf Grundlage dieser Daten ist äußerst aufwändig und ggf. unmöglich. (3) Zumindest in Deutschland gibt es ein ausgeprägtes allgemeines Bewusstsein für Datenschutz sowie ein hohes $\mathrm{Ma} ß$ an technisch-organisatorischen Maßnahmen zum Schutz von (klinischen) Daten (Custers et al. 2018).

Was den potenziellen Nutzen von SeConts betrifft, so ist zwischen dem potenziellen Eigennutzen für datengebende Patient*innen und dem potenziellen Fremdnutzen für zukünftige Patient*innen sowie das Gesundheitswesen insgesamt zu unterscheiden. Ein Eigennutzen für die datengebenden Patient*innen ist sehr unwahrscheinlich. Obwohl SeConts einen starken Praxisbezug aufweist und z. B. Studien zur Arzneimittelsicherheit oder Qualitätsverbesserungsstudien umfasst, dürfte es gewöhnlich für einen Eigennutzen zu lange dauern, bis Kenntnisse aus SeConts in klinisch relevante Veränderungen umgesetzt werden. ${ }^{7}$ Auch ein möglicher Eigennutzen für Patient*innen durch sogenannte Zufalls- oder Zusatzbefunde, d.h. durch gesundheitsrelevante Informationen, welche im Rahmen einer sekundären Datenanalyse außerhalb des Behandlungskontexts entdeckt werden, erscheint im Falle von Se-

\footnotetext{
5 Vgl. hierzu den Kerndatensatz der MII (2017).

6 Vgl. hierzu Saeltzer (2004).

7 Eine Ausnahme könnte eventuell für chronische und nicht stark progredient verlaufende Erkrankungen bestehen, mit denen Patient*innen über mehrere Jahre hinweg leben.
} 
Conts sehr ungewiss. ${ }^{8}$ Aufgrund der geringen Wahrscheinlichkeit für einen Eigennutzen für Patient*innen gehen wir bei den folgenden Überlegungen aus Gründen der Einfachheit und Klarheit der Fragestellung davon aus, dass es kein (ethisch) relevantes Potenzial für Eigennutzen gibt.

Demgegenüber birgt SeConts jedoch ein erhebliches und ethisch relevantes Nutzenpotenzial für ein besseres wissenschaftliches Verständnis von Krankheiten und eine bessere Diagnose und Behandlung zukünftiger Patient*innen. Dies bedeutet nicht, dass jede Form von SeConts notwendigerweise einen maßgeblichen Teil zu dieser Verbesserung beiträgt (vgl. Ploug 2020). Auch zeigen Erfahrungen, besonders aus der Grundlagenforschung, dass Forschungsergebnisse häufig gar nicht und wenn, dann sehr spät eine Auswirkung auf die Behandlung haben (Yarborough 2017). Für ein hohes Nutzenpotenzial von SeConts sprechen jedoch u. a. die folgenden Gründe: (1) SeConts kann in einer Vielzahl verschiedener Studientypen durchgeführt werden und dabei ein sehr breites Spektrum an möglichen Forschungsgebieten abdecken (Jungkunz et al. 2021). (2) Viele Formen von SeConts sind explizit anwendungsbezogen und wurden bereits praktisch durchgeführt (Jungkunz et al. 2021). Die Anwendungsbezogenheit von SeConts garantiert zwar keinen Nutzen, macht diesen aber im Vergleich zu reiner Grundlagenforschung wahrscheinlicher. (3) Die Daten können über einen langen Zeitraum immer wieder in wechselnden Formaten verwendet werden. (4) Die Daten können nicht nur zur Erforschung der Erkrankung der datengebenden Patient*innen verwendet werden, sondern auch für andere Fragestellungen (u. a. als virtueller Kontrollarm). Die Gruppe von Personen, die potenziell von den Daten einer Patientin in SeConts profierten kann, ist somit nicht auf die Gruppe zukünftiger Patient*innen mit der gleichen Erkrankung beschränkt. (4) Besonderes Potenzial besteht für zukünftige Patient*innen mit seltenen Erkrankungen aufgrund der Möglichkeit, durch Aggregation von Datensätzen verschiedener Standorte große Datensätze zu erzeugen. Dieser Punkt ist auch wichtig angesichts der zunehmenden Ausdifferenzierung (Stratifizierung) von Krankheiten (z. B. molekularbasiert in der Onkologie oder generell unter dem Schlagwort der „Personalisierten Medizin“), die durch neue Subtypen zu neuen seltenen Erkrankungen führt.

Damit durchzuführende SeConts das vorhandene Nutzenpotenzial auch wirklich ausschöpfen kann, sollte man vorab gewisse Anforderungen an deren Durchführung stellen, indem z.B. die Einhaltung guter wissenschaftlicher Praxis oder die Vorregistrierung von Studien verlangt werden. Eine weitere Voraussetzung für SeConts und die Ausschöpfung des Nutzenpotenzials besteht darin, dass Kliniken, in denen

\footnotetext{
${ }^{8}$ Gründe hierfür sind: (1) Die Daten wurden bereits von der behandelnden Ärztin im Rahmen der Behandlung befundet. Es ist unwahrscheinlich, dass eine (aggregierte) Analyse der Daten im Rahmen von SeConts neue Erkenntnisse bzgl. des Gesundheitszustands einzelner Patient*innen hervorbringt. (2) Zwischen der Behandlung (und damit der Datenerhebung) und dem Auftreten eines Zufallsbefunds kann zu viel Zeit vergehen, um noch einen Nutzen für datengebende Patient*innen zu erzeugen. (3) Stand jetzt gibt es unseres Wissens keine Erfahrungen zum Vorkommen von Zusatzbefunden aus der Sekundärnutzung klinischer Daten, weshalb sie eine sehr hypothetische Möglichkeit darstellen und nicht als realistisches Nutzenpotenzial gelten können.
} 
die Daten erhoben werden, diese Daten auch wirklich für SeConts zur Verfügung stellen, womit aber gerechnet werden kann. ${ }^{9}$

\section{Mögliche Argumente für eine Pflicht zur Bereitstellung klinischer Daten}

Welche Gründe sprechen für eine Pflicht von Patient*innen, ihre Daten für SeConts bereitzustellen?

\section{Allgemeine Hilfspflicht}

Hier ist zunächst an die allgemeine Hilfspflicht zu denken, nach der jeder die moralische Pflicht hat, Menschen Hilfe zu leisten, wenn sie diese benötigen und die Hilfe dem potenziell Helfenden zumutbar ist. Für das Bestehen einer allgemeinen Hilfspflicht gibt es gute Gründe: Kant betont, dass eine allgemeine Hilfspflicht (Wohltätigkeitspflicht) deshalb existiert, weil eine Ablehnung derselben als allgemeingültige Maxime ein Widerspruch des vernünftigen Willens wäre (Kant 1785, S. 423) und der Zweckformel des kategorischen Imperativs widerspräche (Kant 1785, S. 430). Rawls argumentiert, dass eine allgemeine Hilfspflicht im Urzustand beschlossen würde, weil sie eine positive und ,tiefgreifende Wirkung auf die Qualität des täglichen Lebens“ (Rawls 1979, S. 374) mit sich bringt. Eine besondere Unterart der Hilfspflicht ist die Rettungspflicht. Porsdam Mann et al. (2016) nennen sie als Grund für eine Pflicht von Patient*innen, ihre Daten für SeConts bereitzustellen. Diese Stoßrichtung geht jedoch fehl, weil, wie weiter unten dargelegt werden wird, die Bereitstellung von Daten für SeConts nicht mit einer Rettung gleichgesetzt werden kann. Ungeachtet dieser Ablehnung der Anwendung des Rettungsarguments auf die hier vorliegende Fragestellung informiert jedoch die seit Jahrzehnten andauernde ethische Debatte um globale Hilfs- und Rettungspflichten die hier durchgeführte Analyse der Bedingungen, die für das Vorliegen einer Hilfspflicht erfüllt sein müssen bzw. sich als Faktoren auf die Stärke der Pflicht bzw. den Grad der Verbindlichkeit auswirken (Singer 1972; Bauhn 2011; Miller 2020). Diese Bedingungen werden im Folgenden dargelegt und es wird gezeigt, dass sie im Hinblick auf die hier vorliegende Fragestellung unter bestimmten Voraussetzungen erfüllt sind.

\footnotetext{
9 Von der Annahme, dass Kliniken die Patientendaten für SeConts bereitstellen und nicht systematisch zurückhalten werden, gehen wir aus folgenden Gründen aus: (1) Kliniken erhalten für den Aufbau der Strukturen oft Gelder, müssen sich aber im Gegenzug zur Forschungsweitergabe verpflichten und diese auch nachweisen (vgl. MII). (2) Der wissenschaftliche und politische Trend und Druck zum Datenteilen nimmt seit Jahren zu und dürfte weiter steigen. (3) Über die Herausgabe von Daten entscheidet z. B. im Falle der MII nicht die Klinik- oder Abteilungsleitung, sondern ein Datennutzungs- und Zugangskomitee, in dem eine Verweigerung des Teilens prinzipiell unter Rechtfertigungslast steht. (4) Die mögliche Sorge von Kliniken, dass sie von Extern beforscht werden könnten (ihre Patientenzahlen oder die Versorgungsqualität), kann durch technisch-organisatorische Maßnahmen (Verschleierung des Standortes) und vertragliche Vereinbarungen oder Bedingungen bei „Use and Access“ angemessen adressiert werden.
} 


\section{Bedingung 1: Vorliegen einer Situation der Hilfsbedürftigkeit}

Die allgemeine Hilfspflicht besteht vor allem, wenn die Versorgung einer Person mit Grundgütern mangelhaft oder bedroht ist. Hier wird davon ausgegangen, dass Gesundheit ein Grundgut ist und Krankheiten eine Hilfsbedürftigkeit begründen, auch wenn nicht alle Krankheiten gleichermaßen belastend und bedrohlich sind.

\section{Bedingung 2: Effektivität des möglichen Helfens}

Damit eine mögliche Handlung vorab als Akt des Helfens interpretiert werden kann, muss sie mit einer den Umständen entsprechend angemessen großen Wahrscheinlichkeit einen helfenden Effekt für Hilfsbedürftige mit sich bringen. Dies ist bei SeConts der Fall: Die Bereitstellung klinischer Daten ist mit einer angemessen hohen Wahrscheinlichkeit ein relevanter Beitrag zu SeConts und damit zur Hilfe für zukünftige Patient*innen (zum Nutzenpotenzial von SeConts, vgl. Kapitel Risiken und Nutzen von SeConts). Gegen diese Annahme sind drei mögliche Einwände denkbar: (1) Man könnte anmerken, dass die klinischen Daten von Patient*innen erst dann einen Nutzen bringen, wenn eine gewisse, für die Durchführung von SeConts notwendige ,kritische Masse“ anderer Patient*innen ebenfalls ihre klinischen Daten für SeConts bereitstellt. Somit wäre zu bezweifeln, dass die Bereitstellung der eigenen Daten bereits eine Hilfeleistung darstellt. Dem ist zu entgegnen, dass durch (nationale oder gar internationale) Vernetzung rasch signifikante Kohortengrößen entstehen können und, je nach Art von SeConts, auch wenige Datensätze schon einen Nutzen erzeugen, z. B. bei der Generierung von Hypothesen für zukünftige Forschung. (2) Zusätzlich könnten Kritiker einwenden, es sei ungewiss, dass SeConts zu einer Verbesserung der Versorgung führt, womit auch die Bereitstellung klinischer Daten für SeConts kaum mehr als Hilfeleistung zu werten wäre. Hierauf sind zwei Punkte zu erwidern: (a) In alltäglichen Lebensbereichen gibt es zahlreiche Handlungen, die als Hilfeleistungen gelten, obwohl es nur wahrscheinlich, aber nicht gewiss ist, dass sie tatsächlich helfen (z. B. bei einer kleinen Spende von Geld oder Kleidern für Menschen nach Naturkatastrophen). (b) Bereits im Kapitel Risiken und Nutzen von SeConts wurde das hohe grundsätzliche Nutzenpotenzial von SeConts dargelegt. Selbstverständlich lässt sich vorab nicht bestimmen, wie groß die Bedeutung der klinischen Daten einzelner Patient*innen für zukünftige nützliche Ergebnisse aus SeConts sein wird. Es ist jedoch wahrscheinlich, dass die Daten früher oder später bzw. in der Summe ihrer Verwendungen einen, wenn auch eher kleinen, Beitrag zur Erzeugung von behandlungsrelevantem Wissen leisten werden. Dieser Nutzen kann jedoch nicht als Rettung gelten, da er nicht vergleichbar ist mit einer wirklichen (Not-)Rettung, wie z.B. dem Werfen eines Rettungsrings an einen Ertrinkenden. Das Argument der „Rettung“, wie von Porsdam Mann et al. (2016) mit dem Begriff der Rettungspflicht (duty to easy rescue) vertreten, ist daher für SeConts unangebracht. (3) Man könnte einwenden, dass eine Hilfspflicht nicht gegenüber Personen vorliegen kann, die zum jetzigen Zeitpunkt noch nicht hilfsbedürftig und auch nicht konkret bekannt sind. Dieser Einwand macht deutlich, dass die allgemeine Hilfspflicht im Falle der Bereitstellung klinischer Daten für SeConts nicht die gleiche Dringlichkeit hat, wie in Situationen mit einem konkreten, hilfsbedürftigen Gegen- 
über. In komplexen sozialen Zusammenhängen wie unserer modernen Gesellschaft allgemein sowie dem Gesundheitswesen im Speziellen erstreckt sich die allgemeine Hilfspflicht jedoch über das hilfsbedürftige Gegenüber hinaus auch auf weitere Bereiche wie das Antizipieren zukünftiger Bedürftigkeit. Auch wenn wir nicht wissen, wer in Zukunft krank sein wird, so wissen wir, dass es in der Zukunft Kranke geben wird, die Hilfe benötigen.

\section{Bedingung 3: Zuständigkeit der helfenden Person}

Eine Hilfspflicht besteht für eine Person insbesondere dann, wenn die Person ,zuständig“ ist, insofern die Hilfeleistung nicht ebenfalls von anderen Personen gleichermaßen ausgeführt werden könnte. ${ }^{10}$ In SeConts werden klinische Daten einer großen Zahl von Patient*innen aggregiert, so dass der Beitrag Einzelner auf den ersten Blick unwichtig erscheinen mag. Sicherlich lässt sich, wie bereits erwähnt, vorab nicht mit Gewissheit vorhersehen, ob der Datensatz einer/s Patient*in einen relevanten Beitrag für SeConts leisten wird. Jedoch ist grundsätzlich der Datensatz jedes/jeder einzelnen Patient*in einzigartig und in diesem Sinne bedeutsam, auch wenn er in großen Datensätzen eventuell nur geringes Gewicht entfalten wird. Für den potenziellen Wert jedes einzelnen Datensatzes spricht auch die bereits erwähnte zunehmende Ausdifferenzierung (Stratifizierung) von Krankheiten, wie sie bereits im Kapitel zu Risiken und Nutzen von SeConts erläutert wurde, die jeden Datensatz potenziell wertvoll werden lässt.

\section{Bedingung 4: Zumutbarkeit der Hilfeleistung}

Damit eine Handlung als Gegenstand einer Hilfspflicht gelten kann, darf sie die zuständige Person nicht unverhältnismäßig stark belasten, sondern muss zumutbar sein. ${ }^{11}$ Den ersten wichtigen Aspekt von Zumutbarkeit stellen die Kosten dar, welche die geforderte Hilfsleistung für die hilfeleistende Person mit sich bringt. Hierunter zählen sowohl (a) konkrete Kosten durch den Einsatz von quantifizierbaren Ressourcen (Kraft, Zeit, Geld), als auch (b) Risiken für oder Einschränkungen von nichtquantifizierbaren Werten, Interessen und Rechten, z. B. des Datenschutzes. Um zumutbar zu sein, müssen alle Kosten für die helfende Person in einem angemessenen Verhältnis zu einem zweiten Aspekt stehen: dem potenziellen Nutzen für die hilfsbedürftige Person. Dieser Nutzen setzt sich aus der Wahrscheinlichkeit des Eintretens der intendierten Hilfe und dem Ausmaß (,Größe“ oder „Wichtigkeit“) der Hilfe für die hilfsbedürftige Person zusammen. Was nun die Zumutbarkeit bei SeConts betrifft, so entstehen bei der Bereitstellung von Daten für SeConts in der Regel nur geringe konkrete Kosten für Patient*innen (a), da die Daten im Behandlungs-

\footnotetext{
10 Die Rolle der Zuständigkeit für Vorliegen einer Hilfspflicht wird im Rahmen der Debatte über die Rettungspflichten kontrovers diskutiert und wirft grundlegende ethische Fragen auf (Singer 1972; Bauhn 2011; Miller 2020). Im Rahmen der vorliegenden Arbeit wird Zuständigkeit als verstärkender Faktor für die allgemeine Hilfspflicht interpretiert, nicht als conditio sine qua non für diese Pflicht.

11 Bereits Kant betont, dass die Wohlfahrtspflicht stets unter dem Vorbehalt der Zumutbarkeit steht (Kant 1797, S. 454).
} 
kontext ohnehin erhoben werden. Der Akt der Bereitstellung selbst (in Form der informierten Einwilligung) stellt einen vernachlässigbaren zeitlichen Aufwand für Patient*innen dar. Die möglichen Risiken (b) für Vertraulichkeit und informationelle Selbstbestimmung von Patient*innen wurden bereits als gering eingestuft (siehe Kapitel zu Risiken und Nutzen von SeConts).

Für die Zumutbarkeit entscheidend ist nun, in welchem Verhältnis die Kosten (mit den möglichen Risiken als dem vorrangig relevanten Kostenfaktor) zum potenziellen Nutzen stehen. Die Bewertung der Zumutbarkeit kann hier nur im Rahmen einer groben Einschätzung und Abwägung erfolgen. Dabei kommen wir zu dem Schluss, dass SeConts als zumutbar gelten kann, sofern die Voraussetzungen erfüllt werden, welche im Kapitel Risiken und Nutzen von SeConts erwähnt wurden: ein hohes Datenschutzniveau sowie Maßnahmen zur Ausschöpfung des Nutzenpotenzials, z. B. durch die Einforderung guter wissenschaftlicher Praxis. Im Rahmen einer systematischen Durchführung von SeConts wäre es die Aufgabe einer adäquaten Governance, diese für die Zumutbarkeit relevanten Bedingungen entsprechend zu adressieren und so die Zumutbarkeit für Patient*innen zu gewährleisten.

Fassen wir nun nochmal in Kürze zusammen, inwiefern die für das Vorliegen der allgemeinen Hilfspflicht relevanten Bedingungen gegeben sind: (1) Hilfsbedürftigkeit ist bei Kranken grundsätzlich zu bejahen. (2) Die Effektivität der Hilfeleistung durch die Bereitstellung der klinischen Daten einzelner Patient*innen ist zwar vom Umfang her eher klein, aber dennoch wahrscheinlich und daher letztlich zu bejahen. (3) Die Zuständigkeit der helfenden Person ist ebenfalls aufgrund der Einzigartigkeit jeder Krankheitsgeschichte gegeben. (4) Die Zumutbarkeit ist unter den genannten Bedingungen ebenfalls gegeben. Die allgemeine Hilfspflicht stellt somit einen starken moralischen Grund für eine Pflicht von Patient*innen dar, ihre Daten für SeConts bereitzustellen.

\section{Solidarität}

Im Zusammenhang mit der Frage nach den Pflichten von Patient*innen gegenüber der Gesellschaft wird auch Solidarität als moralischer Grund für eine Mitwirkung an biomedizinischer Forschung genannt (Chadwick und Berg 2001; Hoedemaekers et al. 2007). Um zu klären, ob Solidarität als moralischer Grund für eine Pflicht zur Bereitstellung von Daten in Betracht kommt, müssen zwei Ebenen betrachtet werden. Zunächst ist auf einer grundsätzlichen Ebene der Begriff der Solidarität zu klären und zu fragen, ob das, was unter dem Begriff zu verstehen ist, überhaupt eine Pflicht oder ein moralischer Grund für Pflichten sein kann. Die zweite Ebene betrifft die Frage, ob der Begriff der Solidarität, unter der Annahme, dass er sich als ein moralisch belastbarer Begriff herausstellt, auf die Frage nach einer Pflicht zur Bereitstellung klinischer Daten für SeConts angewendet werden kann. Im Rahmen einer Analyse in gebotener Kürze, die der Komplexität des Begriffs nicht voll gerecht wird, wird im Folgenden auf beiden Ebenen eine kritische Perspektive gegenüber dem Solidaritätsbegriff eingenommen.

Der Begriff der Solidarität hat eine lange und vielfältige Tradition und wird unter anderem in der Soziologie (Durkheim 1992) und der Philosophie (Taylor 1994) sowie in den letzten Jahren auch immer häufiger in der Bioethik behandelt (Prainsack 
und Buyx 2017; Ter Meulen 2017). Außerhalb der akademischen Debatte findet der Begriff auch in der Politik als häufig verwendeter Appell oder gar Kampfbegriff (Bayertz 1998) Anwendung. Wir beziehen uns hier auf den Begriff der Solidarität, wie er in der bioethischen Debatte, gerade auch in Zusammenhang mit Datenbanken, wohl am prominentesten von Prainsack und Buyx vertreten wird. Die beiden Autorinnen verstehen Solidarität als „Praktiken, welche eine Bereitschaft dazu widerspiegeln, Kosten in Kauf zu nehmen, um anderen zu helfen. [...] Diese Bereitschaft [...] basiert regelmäßig auf dem Erkennen von Gemeinsamkeiten mit einer anderen Person [...] in mindestens einer im gegebenen Kontext relevanten Hinsicht" (Prainsack und Buyx 2013, S. 576).

Ein wichtiger Bedeutungsaspekt des Begriffs der Solidarität ist die Bereitschaft, unter Inkaufnahme von Kosten zu helfen. Dieser Aspekt stellt eine deutliche Gemeinsamkeit mit der bereits vorgestellten Hilfspflicht dar. Die obige Bewertung, dass die Hilfspflicht ein starkes Argument ist, steht somit dem Begriff der Solidarität nahe. Die moralische Norm, dass man anderen helfen soll, wird jedoch besser und klarer durch den Begriff der Hilfspflicht ausgedrückt, während der Begriff der Solidarität noch andere Bedeutungsaspekte hat, die offene Fragen aufwerfen. Bereits auf grundsätzlicher Ebene des Begriffs der Solidarität und seines moralischen Gehalts stellen sich folgende Fragen: (1) Der Status des Begriffs der Solidarität ist häufig unklar. Handelt es sich um einen deskriptiven oder einen normativen Begriff? Prainsack und Buyx beispielsweise betonen, dass Solidarität eine soziale Praxis darstellt und sich nicht auf normative Weise auf ein Ideal oder einen Wert bezieht. Sie merken jedoch an, dass Solidarität normative Implikationen haben könne. ${ }^{12}$ (2) Die Verknüpfung von Solidarität mit dem (subjektiven) Wahrnehmen von Gemeinsamkeiten bzw. Ähnlichkeiten zwischen Helfendem und Hilfsbedürftigen ist vor allem bei einer deskriptiven Verwendung intuitiv plausibel und in der Theoriegeschichte der Solidarität, wenngleich in unterschiedlicher Form, weit verbreitet (Durkheim 1992; Taylor 1994). Ein normativ-ethischer Solidaritätsbegriff auf der Basis von Gemeinsamkeiten ist allerdings erklärungsbedürftig. Es gibt verschiedene Aspekte zwischen Menschen, die als relevante Gemeinsamkeiten und damit entscheidender Faktor für solidarisches Handeln wahrgenommen werden können: eine konkrete Notsituation, in der man sich zusammen mit anderen befindet; eine Krankheit; die Zugehörigkeit zu bestimmten Gruppen wie z. B. die der Weißen, der Landwirte oder eines ehemaligen Kampfverbandes. Nicht alle diese Gemeinsamkeiten erscheinen als gute moralische Gründe für ein Gebot der Hilfeleistung. (3) Solidarität, die sich auf Gemeinsamkeiten oder Ähnlichkeiten stützt, ist potenziell ausschließend, antiuniversalistisch und daher ethisch problematisch (Jungkunz 2018). Zwar ist auch ein universalistischer Solidaritätsbegriff denkbar, der sich auf die gesamte Menschheit bezieht. Die relevante Gemeinsamkeit wäre in diesem Fall das „Menschsein“. Ein derart weites Verständnis von Solidarität verweist jedoch eher auf die allgemeine

\footnotetext{
${ }_{12}$ Diese normativen Implikationen würden, so die Autorinnen, zum Beispiel dann zutage treten, wenn eine Handlung, welche nach obiger Definition als solidarisch klassifiziert werden kann, mit hoher Wahrscheinlichkeit einen positiven Effekt für alle Mitglieder der Gesellschaft hat. In diesem Fall sei Solidarität erstrebenswert (Prainsack und Buyx 2017, S. 71). Die Frage nach dem moralischen Gehalt von Solidarität wird allerdings somit auf die Frage nach dem allgemeinen Nutzen bestimmter solidarischer Handlungen ausgelagert.
} 
Hilfspflicht, wie sie im vorigen Abschnitt vorgestellt wurde, da die Begrenzung auf eine bestimmte Gruppe von Personen entfällt.

Obwohl sich schon auf der grundlegenden Ebene offene Fragen ergeben, soll im Folgenden trotzdem noch die potenzielle Anwendbarkeit des Solidaritätsbegriffs auf die in diesem Artikel vorliegende konkrete Fragestellung untersucht werden. Der Bezug auf Solidarität passt auf den ersten Blick gut zum Bereitstellen klinischer Daten durch Patient*innen für SeConts, denn Solidarität stellt ohnehin schon ein de facto anerkanntes politisch-regulatives Kernprinzip des deutschen Gesundheitswesens dar ${ }^{13}$. Bei genauerer Betrachtung zeigt sich jedoch ein gewisser Widerspruch zum Geist des Solidaritätsprinzips im deutschen Gesundheitswesen. Während gemäß dem Prinzip der Solidarität im Gesundheitswesen die Gesunden und Wohlhabenden mit ihren Beiträgen für die Kranken und weniger Wohlhabenden eintreten, würden im Falle der Bereitstellung von Daten für SeConts eher die Kranken, insbesondere die chronisch und schwer Kranken, einen uneigennützigen Beitrag zum Nutzen aller leisten, da im Zuge ihrer Diagnostik und Behandlung in besonders umfangreichem Maße klinische Daten generiert werden. Dies bedeutet, dass die bereits im Kapitel zu Risiken und Nutzen von SeConts angesprochenen - wenngleich geringen möglichen Belastungen und Risiken, welche mit der Menge der erhobenen Daten potenziell steigen, diejenigen besonders betreffen könnten, die ohnehin bereits durch ihre Erkrankung in höherem Maße belastet sind. Dieser mutmaßlich höhere Beitrag chronisch oder schwer erkrankter Patient*innen ist nicht notwendigerweise ethisch problematisch, lässt sich aber mit dem Prinzip der Solidarität, zu dem er in latenter Spannung steht, nicht gut begründen und verweist eher auf das Prinzip des Zurückgebens, welches später näher erläutert wird.

Die offenen Fragen auf der grundlegenden Ebene sowie die Schwierigkeiten bei der Anwendung auf die Fragestellung dieses Artikels legen nahe, den Kerngehalt des Solidaritätsgedankens, das Hilfsgebot, mit dem Argument der allgemeinen Hilfspflicht auszudrücken, und ansonsten darauf zu verzichten, den Begriff der Solidarität im Sinne eines moralisch-normativen Arguments zugunsten einer Pflicht von Patient*innen, ihre klinischen Daten bereitzustellen, zu verwenden.

\section{Pflicht zu gemeinwohlförderlichem Handeln}

Einen weiteren möglichen Grund für eine Pflicht von Patient*innen, ihre klinischen Daten für SeConts bereitzustellen, stellt die Pflicht zu gemeinwohlförderlichem Handeln dar. Die Argumentation mehrerer Autor*innen lässt sich unter eine derartige Pflicht subsumieren: In der Debatte zur Pflicht zur Teilnahme an interventioneller Forschung postulieren Schaefer et al. (2009) eine derartige Pflicht mit der Begründung, dass medizinisches Wissen, welches durch biomedizinische Forschung erzeugt wird, allen in der Gesellschaft nütze. In Bezug auf die Bereitstellung von Daten für die Etablierung eines Lernenden Gesundheitssystems argumentieren Faden et al. (2013) für eine Pflicht von Patient*innen, ihre klinischen Daten für SeConts bereitzustellen, weil SeConts im Interesse aller ist. Ballantyne und Schaefer (2018)

13 https://www.bundesgesundheitsministerium.de/. Zugegriffen: 7. April 2021. 
postulieren eine Pflicht mit Verweis auf den Anspruch der Gesellschaft, die Daten zu nutzen, welche in den Augen der Autor*innen eine ,,public ressource“ darstellen.

Die folgende Argumentation schließt sich unter Rückgriff auf den Begriff des gemeinwohlförderlichen Handelns den Ansätzen der genannten Beiträge an, versucht jedoch auch, etwas näher auf die Hintergründe und Anwendungsbedingungen einzugehen. Die Pflicht zu gemeinwohlförderlichem Handeln ist verwandt mit der allgemeinen Hilfspflicht, bezieht sich jedoch nicht direkt auf Individuen, sondern auf die abstrakte Menge der Allgemeinheit und somit auf komplexe gesellschaftliche Zusammenhänge. Aus diesem Grund spielt bei der Pflicht zu gemeinwohlförderlichem Handeln die Unterstützung von Institutionen zur Förderung des Allgemeinwohls eine große Rolle. Diese Pflicht ist darin begründet, dass der Erhalt gerechter Institutionen für eine gerechte Gesellschaft essenziell ist und ihre Untergrabung jedem auf Dauer schaden würde (Rawls 1979). Gerechte Institutionen sind solche, welche die gerechte Verteilung von sozialen Gütern und Lasten regeln, Rechte sichern und dafür sorgen, dass Bürger*innen zumindest über ein Minimum an Grundgütern verfügen, das notwendig ist, um ein menschenwürdiges Leben zu führen und persönliche Freiheiten auch wirklich nutzen zu können. Das öffentliche Gesundheitswesen kann als gerechte Institution gelten. Etwas anders verhält es sich mit dem Bereich der universitären und außeruniversitären biomedizinischen Forschung, auch wenn er natürlich eng mit dem öffentlichen Gesundheitswesen verbunden ist: Er ist nicht im Sinne sozialer Gerechtigkeit als gerechte Institution anzusehen. Allerdings dient er - u. a. durch seine enge Verbindung zum Gesundheitswesen - auch dem Gemeinwohl, weshalb auch seine Förderung unter die Pflicht zu gemeinwohlförderlichem Handeln subsumiert werden kann.

Damit die Pflicht zu einem bestimmten gemeinwohlförderlichen Handeln vorliegt, müssen, analog zur allgemeinen Hilfspflicht (siehe oben), folgende Bedingungen erfüllt sein: Vorliegen eines legitimen öffentlichen Bedarfs (Interesses); Effektivität der gemeinwohlförderlichen Handlung; Zuständigkeit der gemeinwohlförderlich handelnden Person; Zumutbarkeit der gemeinwohlförderlichen Handlung. All diese Bedingungen sind in Bezug auf die Bereitstellung klinischer Daten für SeConts erfüllt: Die stetige Verbesserung der hochkomplexen öffentlichen Gesundheitsversorgung ist ohne Zweifel im öffentlichen Interesse. Auch wurde bereits erläutert, dass die Beteiligung an SeConts durch die Bereitstellung klinischer Daten einen kleinen aber dennoch effektiven Beitrag zur Verbesserung der medizinischen Versorgung leistet, was auch einen effektiven Dienst am Gemeinwohl darstellt. Über den Nutzen für zukünftige Patient*innen hinaus erstreckt sich der Nutzen einer verbesserten Gesundheitsversorgung (indirekt) auch auf Gesunde, die durch eine hochwertige Gesundheitsversorgung und gesundheitliche Präventionsmaßnahmen profitieren und auch mittelbare Vorteile aus einer ,gesünderen Gesellschaft“ ziehen. Die Bedingung der Zuständigkeit der helfenden Person als ein für den Grad der Verbindlichkeit relevanter Faktor sowie die Bedingung der Zumutbarkeit der Hilfeleistung lassen sich direkt von den obigen Ausführungen zur allgemeinen Hilfspflicht übertragen.

Aus Vorangegangenem folgt, dass das Argument der Pflicht zu gemeinwohlförderlichem Handeln unter den genannten Bedingungen einen starken moralischen Grund für eine Pflicht von Patient*innen darstellt, ihre Daten für SeConts bereitzustellen. 


\section{Das Trittbrettfahrerargument}

Das Trittbrettfahrerargument geht davon aus, dass Personen, die ein öffentliches Gut nutzen, eine moralische Pflicht haben, sich an den Kosten für Produktion oder Erhaltung des öffentlichen Guts zu beteiligen. Wer ein öffentliches Gut nutzt, ohne sich an den Kosten zu beteiligen, ist ein*e Trittbrettfahrer*in und moralisch zu tadeln. Der Begriff des Trittbrettfahrens stammt aus den Wirtschaftswissenschaften und beschreibt dort ein zentrales Problem bestimmter Güter, von deren Nutzung niemand ausgeschlossen werden kann (Nicht-Ausschließbarkeit). Kann ein solches Gut von mehreren Personen gleichzeitig genutzt werden, spricht man von einem nicht-rivalen und damit von einem öffentlichen Gut (Sturn 2020). Ist eine Nutzung durch mehrere ohne Einbußen der Nützlichkeit nicht möglich, spricht man von einem Allmendegut (Rohland 2021). Durch die Nicht-Ausschließbarkeit ist es im Sinne der Eigennutzenmaximierung für jeden Einzelnen ,wirtschaftlich rational“", das Gut zu nutzen, ohne sich an den Kosten seiner Produktion oder Aufrechterhaltung zu beteiligen. Diese „Rationalität“" verhindert oder bedroht jedoch die Produktion bzw. das Fortbestehen des Guts selbst.

Man könnte nun fragen, ob das Trittbrettfahrerargument überhaupt als ein eigenständiges Argument gelten kann oder ob es vielmehr nur eine Unterform der Pflicht zu gemeinwohlförderlichem Handeln ist, schließlich geht es doch bei beiden um Güter des Gemeinwesens. Ein wichtiger und eindeutiger Unterschied zur Pflicht zu gemeinwohlförderlichem Handeln besteht jedoch darin, dass ein ethisch wesentliches Element des Trittbrettfahrerarguments in der Nutzung eines Guts bzw. im persönlichen Profitieren von einem Gut liegt, während das Nutzen bzw. Profitieren keine Voraussetzung für die Geltung der Pflicht zu gemeinwohlförderlichem Handeln ist. Das Trittbrettfahrerargument scheint sich zudem auf eine spezielle Art von gemeinsamen Gütern zu beziehen, nämlich öffentliche und Allmendegüter.

In der Debatte um eine mögliche Pflicht zur Teilnahme an interventioneller Forschung wird argumentiert, dass Patient*innen vom öffentlichen Gut medizinischer Forschung profitieren und daher, um keine Trittbrettfahrer*innen zu sein, die Pflicht haben, sich an medizinischer Forschung zu beteiligen (Harris 2005; Miller 2008; Rhodes 2010). Angewandt auf die Fragestellung dieses Artikels würde das Trittbrettfahrerargument wie folgt lauten: Patient*innen haben die Pflicht, ihre klinischen Daten für SeConts bereitzustellen, da Patient*innen ein öffentliches Gut nutzen und andernfalls Trittbrettfahrer*innen sind. Das Trittbrettfahrerargument erscheint auf den ersten Blick sowohl auf der Ebene seiner grundsätzlichen ethischen Geltung als auch auf der Ebene seiner möglichen Anwendung auf die Fragestellung dieses Artikels plausibel. Bei genauerer Betrachtung zeigen sich jedoch offene Fragen auf beiden Ebenen.

\section{Grundsätzliche Fragen zum Trittbrettfahrerargument}

Es scheint intuitiv nahe zu liegen, Trittbrettfahren aus dem deskriptiv-analytischen Modell der Wirtschaftswissenschaften herauszulösen, moralisch zu verurteilen und daraus ein ethisches Argument abzuleiten. Bei näherer Betrachtung stellen sich aber bei einer Übertragung in die Angewandte Ethik grundsätzliche Fragen, die nicht 
ausreichend bedacht und geklärt sind. Hiermit ist nicht gesagt, dass das Trittbrettfahrerargument aufgrund seiner Herkunft in den Wirtschaftswissenschaften per se keine valide ethische Bedeutung haben kann. Es ist allerdings zu klären, ob und inwiefern der Hintergrund und die spezielle sozial-ökonomische Problematik, die wirtschaftswissenschaftlich mit dem Begriff des Trittbrettfahrens impliziert ist, eventuell auch eine ethische Rolle spielen. Welche ethische Bedeutung wird z.B. den speziellen sozial-ökonomischen Umständen zugemessen, die eine Dynamik bis hin zur Bedrohung der Existenz des öffentlichen Guts entfalten können? Misst man den speziellen ökonomischen Aspekten eine ethische Bedeutung bei, stellt sich die Anschlussfrage: Lassen sich die Kriterien der Nicht-Ausschließbarkeit und der Nicht-Rivalität (mehr oder weniger gut) auf das öffentliche Gesundheitswesen als reelles Guts anwenden und ist das Gesundheitswesen entsprechend als ein öffentliches Gut (oder ein Allmendegut oder ein anderes Gut) anzusehen? Weitere zu klärende Aspekte, die weniger von den speziellen ökonomischen Zusammenhängen herrühren, betreffen zum einen die Frage, ob eine Vereinbarung oder eine irgendwie legitimierte vorausgehende Entscheidung der Gemeinschaft, etwas zu einem öffentlichen Gut oder einem Allmendegut zu machen, erforderlich ist, damit bei Nutzung ein Beitrag dazu moralisch geboten ist. Zudem erscheint klärungsbedürftig, worin genau die normativ-ethischen Elemente bestehen, die Trittbrettfahren unmoralisch machen (Arneson 1982; Cullity 1995).

\section{Anwendbarkeit des Trittbrettfahrerarguments auf SeConts}

Die aufgezeigten offenen Fragen sowie weitere zeigen sich auch mit Blick auf eine mögliche Anwendung des Trittbrettfahrerarguments auf die Fragestellung dieses Artikels: (1) Patient*innen in Deutschland sind gezwungenermaßen krankenversichert und somit zwangsläufig Nutznießer des Guts ,evidenzbasiertes Gesundheitssystem“. Es ist jedoch eine weitere offene grundsätzliche Frage, ob eine Pflicht zur Beteiligung an der Produktion eines Guts mit Verweis auf die persönliche Nutzung gerechtfertigt ist, wenn der Nutznießer keine Möglichkeit hat, das Gut nicht zu nutzen (Shapshay und Pimple 2007). Zwar könnte mit Blick auf SeConts jede*r Bürger*in sich stets darum bemühen, die ihm qua Zwangsmitgliedschaft im öffentlichen Gesundheitssystem zustehende Versorgung im Krankheitsfall möglichst nicht zu nutzen. Dies scheint aber keine faire Wahl darzustellen und kaum zumutbar zu sein. (2) Patient*innen (bzw. Bürger*innen) nutzen das öffentliche Gesundheitssystem nicht ohne Gegenleistung (Brassington 2007): Sie erbringen Krankenkassenbeiträge und finanzieren die öffentliche biomedizinische Forschung mit einem Teil ihrer Steuern und können daher für die Nutzung des Gesundheitswesens nicht pauschal als Trittbrettfahrer*innen bezeichnet werden. Könnten sie aber mit Blick auf die Nutzung klinisch relevanter Erkenntnisse, die aus SeConts stammen, als Trittbrettfahrer*innen bezeichnet werden, so dass daraus ein Argument für ihre Pflicht, an SeConts teilzunehmen, entsteht? Zur Beantwortung dieser Frage sind zwei weitere Fragen zu beantworten, die zumindest in diesem Artikel nicht ohne Weiteres geklärt werden können: (a) Profitieren Patient*innen jetzt schon in relevantem Umfang von Erkenntnissen aus SeConts? Dies kann aus unserer Sicht nicht als generell zutreffend angenommen werden und müsste weiter geklärt werden, was aber schwierig ist. 
(b) Es stellt sich die generelle Frage, in welcher „Währung“ gemäß des Trittbrettfahrerarguments ein angemessener Beitrag zu leisten ist. Kann man seinen Beitrag mit Geld erbringen oder muss man einen Beitrag in den Währungen bzw. in Form von Leistungen und Dingen erbringen, die für die Schaffung bzw. Aufrechterhaltung des Guts wesentlich sind? Müssen Patient*innen, die relevant von Erkenntnissen aus interventionellen klinischen Studien profitieren, selbst auch an klinischen Studien teilnehmen, um keine Trittbrettfahrer*innen zu sein - oder reicht es, wenn sie klinische Studien finanziell unterstützen? (3) Zum jetzigen Zeitpunkt gibt es weder eine gesellschaftliche Vereinbarung oder Regel noch ist es gelebte Praxis, dass alle „Nutzer" des evidenzbasierten Gesundheitssystems einen Beitrag durch die Bereitstellung ihrer klinischen Daten leisten. Es ist auf grundsätzlicher Ebene bisher nicht geklärt, ob eine Vereinbarung oder gelebte Praxis, denen zufolge alle Nutzer eines definierten Guts zu dessen Schaffung und Erhaltung beitragen müssen, für die Gültigkeit des Trittbrettfahrerarguments ethisch relevant oder gar grundlegend ist. Ein relevanter ethischer Aspekt des Trittbrettfahrerarguments könnte nämlich darin gesehen werden, dass Trittbrettfahrer*innen das Gut nutzen, sich aber im Gegensatz zu den anderen selbst nicht an die abgemachte Vereinbarung halten, dass sie einen Vertrauensbruch begehen und dass sie sich (dadurch) eine unzulässige Vorzugsbehandlung (Cullity 1995) gewähren. So lange diese Frage nicht geklärt ist, bleibt auch die ethische Relevanz dieses Aspekts bei der Anwendung des Trittbrettfahrerarguments auf unsere Fragestellung unterbestimmt. Sollte jedoch in Zukunft die Bereitstellung klinischer Daten für SeConts die gesellschaftlich anerkannte Regel werden, z. B. im Rahmen der systematischen Weiterentwicklung der klinischen Versorgung im Sinne eines Learning Health Care Systems, so würde diese Schwachstelle des angewandten Trittbrettfahrerarguments wegfallen.

Auch wenn das Trittbrettfahrerargument intuitiv zunächst plausibel erscheint, so zeigt sich, dass es sowohl auf grundsätzlicher Ebene als auch in Bezug auf die Anwendung für die Fragestellung dieses Artikels wichtige, schwer zu klärende bzw. bisher ungeklärte Fragen aufwirft und daher zum jetzigen Zeitpunkt keinen überzeugenden Grund für eine Pflicht von Patient*innen darstellt, ihre Daten für SeConts bereitzustellen.

\section{Transgenerationale Gerechtigkeit}

Ein weiterer möglicher Grund für eine Pflicht von Patient*innen, sich durch die Bereitstellung ihrer klinischen Daten an SeConts zu beteiligen, kann aus der Debatte um eine mögliche Pflicht zur Teilnahme an interventionellen Studien gewonnen werden und soll im Folgenden als ,transgenerationale Gerechtigkeit“ bezeichnet werden. In der Debatte um eine Teilnahmepflicht an interventionellen Studien gehen einige Bioethiker davon aus, dass Patient*innen im Moment ihrer Behandlung immer von Forschung profitieren, die durch Mitwirkung früherer Patient*innen oder Proband*innen ermöglicht wurde, und leiten aus diesem Umstand eine Pflicht heutiger Patient*innen ab, einen Beitrag für die Forschung zu leisten, um so ihrerseits zukünftigen Generationen von Patient*innen zu helfen (Evans 2004; Harris 2005; Orentlicher 2005). Übertragen auf die Fragestellung dieses Artikels besagt das Argument, dass heutige Patient*innen als Nutznießer*innen vergangener Forschung 
die Pflicht haben, sich durch die Bereitstellung ihrer klinischen Daten an SeConts zu beteiligen, um zukünftigen Patient*innen zu helfen.

Zunächst steht außer Frage, dass Patient*innen heute davon profitieren, dass Patient*innen in der Vergangenheit an Forschung teilgenommen haben. Das Argument der transgenerationalen Gerechtigkeit spricht auch eine wichtige Dimension der Identität und des Selbstverständnisses von Menschen an: dass wir uns als Teil einer historisch-kulturellen Überlieferung und eines größeren sozialen und generationsübergreifenden Zusammenhangs betrachten. Anders als bei ähnlichen Prinzipien, wie dem der Nachhaltigkeit oder der Gerechtigkeit für zukünftige Generationen geht es bei der transgenerationalen Gerechtigkeit nicht darum, eine Ressource unbeschadet zu überliefern bzw. zukünftigen Generationen keine Kosten aufzubürden, sondern um eine Verbesserung (der Sicherheit, Effektivität und Wirtschaftlichkeit von Diagnosen und Therapien) für zukünftige Patient*innen und die Allgemeinheit.

Eine Pflicht heutiger Patient*innen, durch die Bereitstellung ihrer klinischen Daten für SeConts an einer Verbesserung mitzuwirken, wird im Rahmen des Arguments der transgenerationalen Gerechtigkeit mit vergangenen Handlungen früherer Generationen begründet. Diese Begründung hat zwei Schwachstellen: (1) Es stellt sich die komplexe ethische Frage, welche Eigenschaften es konkret sind, die vergangene Handlungen derart moralisch relevant werden lassen, dass sie heutigen Generationen eine Pflicht auferlegen. Schließlich profitieren wir heute von vielen Handlungen vergangener Generationen, die nicht alle eine Pflicht mit sich bringen (Brassington 2007). Die Absicht bzw. Motivation, mit der eine Handlung ausgeführt wurde, kann als eine solche moralisch relevante Eigenschaft betrachtet werden, beispielsweise, wenn die Teilnahme an Forschung durch vergangene Probanden aus dem altruistischen Motiv heraus geschah, die Versorgung zukünftiger Patient*innen $\mathrm{zu}$ verbessern. Heute, aus der Retrospektive, ist es allerdings nicht möglich festzustellen, ob und in welchem Umfang sich frühere Studienteilnehmer*innen von altruistischen Motiven leiten ließen oder eher vom Erhalt einer Aufwandsentschädigung oder der (unberechtigten) Hoffnung auf einen therapeutischen Eigennutzen. (2) Es besteht auch Klärungsbedarf bezüglich der Frage, wem gegenüber heutige Generationen von Patient*innen eine Pflicht haben (Pflichtadressat). Das Argument der transgenerationalen Gerechtigkeit beschreibt keine Pflicht, die unabhängig von vorherigen Handlungen besteht (wie z. B. die allgemeine Hilfspflicht), sondern eine Verpflichtung, wie sie gewöhnlich im Binnenverhältnis zweier Personen oder Parteien entsteht: Wenn z. B. Person A Person B einen Gefallen tut, dann ist B gegenüber A zu Dank verpflichtet. Bei genauerer Betrachtung zeigt sich jedoch, dass beim Argument der transgenerationalen Gerechtigkeit ein komplizierteres Verhältnis zwischen den Akteuren vorliegt: Die moralisch relevanten Handlungen wurden von früheren Patient*innen und Studienteilnehmer*innen X ausgeübt. Diejenigen, die von der Bereitstellung klinischer Daten heutiger Patient*innen durch SeConts profitieren sollen, sind zukünftige Patient*innen Z. Schulden heutige Patient*innen Y die Studienteilnahme den früheren Patient*innen und Studienteilnehmer*innen X, so ist unklar, wie diese Schuld durch die Bereitstellung von Daten für SeConts zugunsten zukünftiger Patient*innen Z ,beglichen“ werden kann. Schulden jedoch heutige Patient*innen Y den zukünftigen Patient*innen $\mathrm{Z}$ eine Beteiligung an $\mathrm{Se}$ - 
Conts, so ist unklar, wie diese Schuld in Handlungen früherer Patient*innen und Studienteilnehmer*innen X begründet sein kann.

Diese Kritik ist nicht so zu verstehen, als gäbe es keine moralische Pflicht, durch die Bereitstellung klinischer Daten für SeConts zukünftige Patient*innen zu unterstützen. Die transgenerationale Gerechtigkeit mit dem Verweis auf Handlungen früher Patient*innen stellt hierfür jedoch keinen starken Grund dar und kann höchstens die Rolle einer motivationalen Grundlage oder eines sinnstiftenden Narrativs für die Bereitstellung klinischer Daten für SeConts spielen.

\section{Das Prinzip des Zurückgebens}

Das Prinzip des Zurückgebens besagt, dass diejenigen, die eine Leistung in Anspruch nehmen oder von einer Institution profitieren, etwas zurückgeben sollten, sofern dafür Gelegenheit und Bedarf bestehen und es ohne Weiteres zumutbar ist. Wenn jemand als Kind und Jugendlicher von einem Sportverein profitiert hat, ist es moralisch geboten, dass die Person diesem Verein im Erwachsenenalter etwas zurückgibt, wenn dies mit geringem Aufwand und geringen Kosten möglich ist. Übertragen auf die Fragestellung dieses Artikels besagt das Prinzip des Zurückgebens, dass es für diejenigen, die eine zufriedenstellende bzw. angemessene Leistung des Gesundheitssystems empfangen, moralisch geboten ist, bei passenden Umständen etwas an das Gesundheitssystem zurückzugeben, insbesondere wenn es sich um eine eher anspruchsvolle und umfangreiche Leistung handelt wie z. B. bei schweren oder chronischen Erkrankungen.

In Gegensatz zur allgemeinen Hilfspflicht und zur Pflicht zu gemeinwohlförderlichem Handeln setzt das Prinzip des Zurückgebens, ähnlich dem Trittbrettfahrerargument, voraus, dass Patient*innen einen Nutzen hatten bzw. eine bestimmte Leistung empfangen haben. Im Gegensatz zum Trittbrettfahrerargument wird jedoch beim Prinzip des Zurückgebens keine bestimmte Form des genutzten Guts (öffentliches oder Allemendegut) vorausgesetzt. Auch dient das Zurückgeben nicht notwendigerweise dem Erhalt des genutzten Guts selbst. Das Prinzip des Zurückgebens unterscheidet sich auch von einer Reziprozität im Sinne eines quid pro quo, wie sie Cohen (2018) anführt, wenn er eine Pflicht von Patient*innen, ihre klinischen Daten für SeConts bereitzustellen, damit begründet, dass sie mehr oder weniger direkt von SeConts profitieren. Das Prinzip des Zurückgebens setzt nicht voraus, dass Patient*innen speziell von SeConts profitieren, sondern nur, dass sie eine zufriedenstellende Leistung des Gesundheitssystems erhalten haben.

Mit dem Prinzip des Zurückgebens soll die Verteilung der finanziellen Kosten gemäß des sogenannten Solidaritätsprinzips des deutschen Gesundheitswesens in keiner Weise angetastet werden. Eine Verknüpfung von Krankheit bzw. Krankheitsrisiko und der Höhe einer finanziellen Beteiligung (z. B. in Form der Krankenkassenbeiträge) ist aus Gerechtigkeitsgründen abzulehnen. Ebenso sollen Patient*innen nicht für ihre Krankheit verantwortlich gemacht werden. Jedes Mitglied der Solidargemeinschaft hat ein Recht auf die Leistungen des Gesundheitssystems und bleibt demselben auch keine Rückzahlung über die geleisteten Krankenkassenbeiträge hinaus schuldig. Das Prinzip des Zurückgebens kommt vielmehr auf einer moralisch weniger verbindlichen Ebene vor dem Hintergrund ins Spiel, dass das 
Gesundheitswesen als Ganzes aufgrund von Überbelastungen und Ressourcen- und Finanzknappheit und in Anbetracht des teilweise erheblichen und kontinuierlich steigenden Ausmaßes an Kosten und Aufwand vieler Behandlungen nur gut und zukunftsfähig funktionieren kann, wenn jede*r mehr beiträgt, als es die rechtlichen Pflichten verlangen. In Anbetracht dieser Umstände sollten Patient*innen etwas zurückgeben, was ihnen zumutbar ist, sie nicht finanziell belastet und kein Potenzial hat, sozioökonomische Ungleichheiten zu verstärken, aber für das Gesundheitswesen dennoch hilfreich ist.

Durch die Bereitstellung klinischer Daten für SeConts etwas zurückzugeben bietet sich der Sache nach an. Dafür spricht auch, dass man eine gewisse Proportionalität zwischen dem Umfang der erzeugten Daten und dem Umfang der in Anspruch genommenen Behandlung erwarten kann. Von Patient*innen, die aufgrund schwerer oder chronischer Erkrankungen das Gesundheitssystem in besonderem Maße in Anspruch nehmen, wird in der Regel eine besonders große Menge an klinischen Daten erzeugt. Die Bereitstellung der Daten für SeConts ist somit eine Möglichkeit, etwas grob proportional zur Inanspruchnahme des Gesundheitssystems und unabhängig vom eigenen finanziellen Status an das Gesundheitssystem zurückzugeben. Die Bereitstellung ihrer Daten für SeConts ist, sofern aufgrund von Datenschutzund Governance-Maßnahmen mit nur sehr geringen Risiken verbunden, eine sehr geringe zusätzliche Belastung für Patient*innen, aber ein potenziell wertvoller und daher naheliegender Beitrag im Sinne des Zurückgebens.

Es lässt sich festhalten, dass das Prinzip des Zurückgebens zwar keinen starken Grund für eine Pflicht von Patient*innen darstellt, ihre klinischen Daten für SeConts bereitzustellen, aber unter bestimmten Bedingungen ein schwacher Grund und eine zusätzliche Motivation sein kann, gerade dann, wenn die erhaltene Behandlung komplex war und zur Zufriedenheit durchgeführt wurde.

\section{Das Prinzip des Nicht-Schädigens}

Das Prinzip des Nicht-Schädigens ist fester Bestandteil der Professionsethik von Ärzt*innen (in Form des „primum non nocere“), der Bioethik, der allgemeinen Ethik und auch der Alltagsethik. In der Debatte um die Teilnahme an interventionellen Studien wird mit Rekurs auf das Prinzip des Nicht-Schädigens argumentiert, dass Patient*innen an Forschung teilnehmen sollen, um Schaden durch die NichtVerbesserung der Versorgung für zukünftige Patient*innen zu vermeiden (Harris 2005). Das Argument setzt das Unterlassen einer Handlung, die dazu geeignet ist, die Versorgung zukünftiger Patient*innen zu verbessern, mit dem aktiven Zufügen von Schaden gleich. Dies ist per se falsch. Stellen wir uns einen „extremen“ Fall vor, in dem einer Patientin durch einen sogenannten Heilversuch das Leben gerettet wird und sich die Frage stellt, ob die Daten aus der Behandlung weitergeben werden. Diese Patientin hat eine moralische Pflicht, die Daten aus diesem Heilversuch für SeConts bereitzustellen, wenn diese Bereitstellung für zukünftige Patient*innen in der gleichen Lage ebenso lebensrettend sein könnte. Die Begründung für diese Pflicht ist jedoch nicht, dass die Patientin durch das Ablehnen der Datenbereitstellung andere Patient*innen schädigen würde, sondern vielmehr ihre Pflicht zu helfen, was wiederum auf die allgemeine Hilfspflicht verweist. 
Aus genannten Gründen ist die Argumentation über das Prinzip des Nicht-Schädigens sowohl in seiner Anwendung auf die Frage der Teilnahme an interventionellen Studien als auch bezüglich seiner Anwendung auf die Frage nach moralischen Gründen für eine Pflicht von Patient*innen, ihre Daten für SeConts bereitzustellen, nicht überzeugend.

\section{Forschungsfreiheit und der Wert von Wissenschaft}

Nachdem die bisher untersuchten Argumente stets die Patient*innen in ihrem Verhältnis zur Gesellschaft im weiteren Sinne im Blick hatten, wird nun das Verhältnis von Patient*innen zu Forschenden näher untersucht. Diese Untersuchung geschieht unabhängig von der Frage, ob und inwiefern Forschung einen gesellschaftlichen Nutzen erzeugt, da der Aspekt des gesellschaftlichen Nutzens letztlich wieder auf das Verhältnis von Patient*innen zur Gesellschaft verweisen würde.

Zunächst ist zu prüfen, ob das Grundrecht der Forschungsfreiheit von Forscher*innen einen Anspruch auf die Nutzung klinischer Daten von Patient*innen begründen kann. Auf einer Ebene der elementaren liberalen Bestimmung der Verhältnisse zwischen Bürger*innen im Anschluss an Rawls' (1979) konsequente Auslegung der Prinzipien der Gleichheit und Freiheit als zentralen Prinzipien der Aufklärung, sollen alle Bürger*innen das gleiche und größtmögliche System an Freiheiten haben, das mit dem System an Freiheiten aller anderen Bürger*innen vereinbar ist. Wenn nun die Ausübung der Forschungsfreiheit (d.h. das Forschen) die Grundfreiheit bzw. das Grundrecht von Bürger*innen auf informationelle Selbstbestimmung berührt, so findet die Forschungsfreiheit an jener Stelle ihre Begrenzung. Die Forschungsfreiheit erlaubt aus ethischer Sicht prima facie keinen Eingriff in die informationelle Selbstbestimmung der Bürger*innen und kann keine ausreichende ethische Rechtfertigung für einen Anspruch von Forscher*innen auf Bereitstellung der klinischen Daten seitens der Bürger*innen (Patient*innen) darstellen.

Vielleicht gibt es jedoch andere moralische Gründe dafür, dass Bürger*innen Forscher*innen bei der Forschung aktiv unterstützen sollen, solange dies für Bürger*innen zumutbar ist. Ein möglicher Grund besteht in dem besonderen Wert, der im Rahmen des liberal-demokratischen Wertesystems der Wissenschaft und ihren Idealen der Objektivität und offenen und kritischen Suche nach Erkenntnis zugesprochen wird. Der Wissenschaft kommt eine Wertdimension ,um ihrer selbst willen“ zu, die unabhängig von etwaiger erwünschter oder erstrebter sozialer Nützlichkeit der Wissenschaft ist. Bürger*innen unterstützen (akademisch-öffentliche) Forscher*innen bereits mit einem kleinen Prozentsatz ihrer Steuern, der für die Finanzierung öffentlicher Forschung verwendet wird. Dass Bürger*innen den Forscher*innen darüber hinaus - und gänzlich unabhängig von einem potenziellen gesellschaftlichen Nutzen - noch weitere Unterstützung schulden, erscheint jedoch zweifelhaft.

Zusammenfassend ist festzuhalten, dass die Bereitstellung klinischer Daten zwar eine Unterstützung für die Wissenschaft und die Verwirklichung der Forschungsfreiheit darstellt, dass aber weder die Forschungsfreiheit noch der Wert der Wissenschaft einen moralisch verbindlichen Anspruch der Forscher*innen auf Nutzung der klini- 
schen Daten von Patient*innen bzw. eine Pflicht von Patient*innen, ihre klinischen Daten für SeConts bereitzustellen, rechtfertigen.

\section{Ergebnis}

Nach der Analyse einzelner möglicher Gründe für eine Pflicht von Patient*innen, ihre klinischen Daten für SeConts bereitzustellen, sollen zum Abschluss die ethischen Ergebnisse konkretisiert werden. Dazu werden Antworten auf zwei Fragen formuliert, von denen die erste unmittelbar den Fokus des Artikels widerspiegelt und die zweite ein Weiterdenken der Antwort auf die erste Frage darstellt:

1. Wie gut bzw. stichhaltig und gewichtig sind die genannten Gründe, die für die Rechtfertigung einer Pflicht von Patient*innen, ihre Daten zu SeConts bereitzustellen, in Betracht kommen?

2. Was folgt summa summarum aus der Betrachtung der pro und contra Gründe mit Blick auf die Rechtfertigung einer Pflicht?

Ad (1): Es wurde gezeigt, dass das Prinzip des Nicht-Schädigens und die Forschungsfreiheit und der Wert der Wissenschaft keine geeigneten Gründe für diese Pflicht darstellen. Die Argumente der Solidarität und des Trittbrettfahrens werfen zu viele ungeklärte Fragen auf und scheinen daher ebenfalls nicht überzeugend. Das Argument der transgenerationalen Gerechtigkeit und das Prinzip des Zurückgebens können als schwache moralische Gründe für eine Pflicht gesehen werden. Sie sind wichtige motivationale Faktoren, ihnen entspricht jedoch keine berechtigte moralische Erwartung von Seiten Dritter darauf, dass Patient*innen ihre Daten für SeConts zur Verfügung stellen. Die allgemeine Hilfspflicht und die Pflicht zu gemeinwohlförderlichem Handeln hingegen stellen starke Gründe für eine moralische Pflicht von Patient*innen dar, ihre klinischen Daten für SeConts bereitzustellen. Sie haben das Potenzial, entsprechende Erwartungen seitens der Gesellschaft bzw. zukünftiger Patient*innen und letztlich eine Pflicht der Patient*innen zu rechtfertigen.

Die ethische Bewertung der zwei Argumente als starke Gründe hängt allerdings entscheidend an der Einschätzung dreier zentraler faktischer Sachverhalte: Wir kommen zu der Einschätzung (a), dass die informationellen Risiken für die Patient*innen unter den genannten Bedingungen (Datenschutzmaßahmen) im Vergleich zur reinen ärztlich-medizinischen Versorgung mit den dabei gewöhnlich genutzten digitalen Instrumenten wie z.B. elektronischen Dokumentationssystemen nicht signifikant erhöht sind. Wir kommen außerdem zu der Einschätzung (b), dass das generelle Nutzenpotenzial von SeConts hoch ist und (c), dass der Datensatz jeder einzelnen Patientin zur Entfaltung des potenziellen Nutzens von SeConts grundsätzlich einen zwar kleinen, aber der Sache nach relevanten Beitrag leisten kann. Die Einschätzungen zu den drei Sachverhalten ( $\mathrm{a}, \mathrm{b}$ und $\mathrm{c}$ ) sprechen alle für die beiden Gründe allgemeine Hilfspflicht und Pflicht zu gemeinwohlförderlichem Handeln und lassen sie daher besonders plausibel und gewichtig erscheinen. Die Einschätzungen der drei Sachverhalte haben jedoch keine starke empirische Grundlage in Form von Erfahrungswerten. Dies ist eine - aus unserer Sicht nicht vermeidbare - Schwachstelle 
unserer Analyse und somit auch der zwei als stark bewerteten Gründe, die für eine Pflicht von Patient*innen sprechen.

Ad (2): Um nun dieses Ergebnis weiterdenkend auf die Frage anzuwenden, ob die genannten Gründe eine Pflicht von Patient*innen rechtfertigen, sind selbstverständlich auch die Argumente in Betracht zu ziehen, die gegen eine solche Pflicht sprechen. Zwar lag der Fokus des Artikels bisher auf der Analyse möglicher Argumente für eine Pflicht. Dabei darf aber nicht übersehen werden, dass diese Analyse durchaus kritisch war und in ihrem Verlauf einige mögliche Gründe für eine Pflicht ganz verworfen und andere als schwach eingestuft wurden. Die Anzahl möglicher Gründe gegen eine Pflicht ist deutlich geringer und diese Gründe sind auch weniger klärungsbedürftig. Ein zentraler möglicher Grund gegen eine Pflicht besteht in den informationellen Risiken für Patient*innen. Diese wurden im Kapitel Risiken und Nutzen von SeConts dargelegt und als einzig beachtenswerte Risiken für Patient*innen eingestuft, aber als sehr gering eingeschätzt. Neben diesem patientenzentrierten Grund gegen eine Pflicht könnten auch die Einschätzungen zu den Sachverhalten b und c gegen eine Pflicht sprechen, wenn sie anders ausfallen würden. Würde man die Nutzenrelevanz des einzelnen Datenbeitrags wesentlich geringer einschätzen und/oder das Nutzenpotenzial von SeConts nur als gering betrachten, würde die Bereitstellung von Daten keinen hilfreichen Beitrag mehr darstellen. Damit wären zentrale Bedingungen des Hilfspflichtarguments und des Arguments der Pflicht zu gemeinwohlförderlichem Handeln nicht mehr gegeben und diese beiden als stark bewerteten Argumente sehr geschwächt.

Akzeptieren wir die Einschätzungen ( $a, b, c)$, wie sie oben getroffen wurden, die allesamt zugunsten einer Pflicht ausfallen und die anderslautend auch Argumente gegen eine Pflicht darstellen würden, dann kommen wir zu dem Schluss, dass es eine moralische Pflicht von Patient*innen gibt, ihre Daten für SeConts bereitzustellen, und dass es einen entsprechenden berechtigten moralischen Anspruch seitens Dritter (der Gesellschaft und zukünftiger Patient*innen) gibt. Hier ist auf zwei Vorbehalte hinzuweisen: (i) Auch im Fall, dass die Einschätzung der zentralen Sachverhalte korrekt ist, kann es in Einzelfällen dazu kommen, dass gewichtige entgegenstehende ethische Gesichtspunkte die Pflicht überwiegen. So können einzelne Patient*innen einem individuell erhöhten Risiko durch SeConts ausgesetzt sein, weil ihre Daten besonders sensibel sind oder ihre Daten sensible Aussagen über ihre Verwandten ermöglichen und somit auch für diese ein Risiko darstellen. Mit Blick auf derartige mögliche Szenarien ist die Pflicht, Daten für SeConts zur Verfügung zu stellen, als eine prima facie Pflicht (Ross 2002 [1930]) zu verstehen, welche in einer bestimmten Situation zwar prinzipiell gilt, aber eventuell hinter anderen in der Situation entgegenstehenden moralischen Gesichtspunkten zurückstehen muss. ${ }^{14}$ (ii) Man sollte angesichts der schwachen empirischen Grundlagen, mit denen die Einschätzung in den drei wichtigen Sachverhalten getroffen wurden (siehe voriger Absatz), in einigen Jahren eine Neubewertung dieser Sachverhalte unter Hinzuziehung bis dahin neu hinzugekommener Erfahrungswerte durchführen. Sofern diese Neubewertung in ethisch relevantem Maße andere Einschätzungen nahelegt, sollte entsprechend auch die ethische Bewertung angepasst werden.

$14 \mathrm{Zu}$ diesem Verständnis von prima facie Pflichten siehe auch Frankena (1972) und Birnbacher (2007). 
Betrachten wir nun zum Ende noch kurz die potenzielle Relevanz der Ergebnisse. Die Pflicht besagt, dass Patient*innen, die nach einer Einwilligung für die Nutzung ihrer klinischen Daten in SeConts gefragt werden, diese Einwilligung aus moralischen Gründen erteilen sollen, so dies in oben erläutertem Sinne zumutbar ist. Intuitiv könnte man hier annehmen, dass ,dieses Sollen“ die Freiwilligkeit der Entscheidungssituation verletzt. Es sei jedoch darauf hingewiesen, dass das moralische Sollen nicht in Widerspruch zur Freiwilligkeit steht. Freiwilligkeit wird in der Regel als Abwesenheit von äußeren und inneren Zwängen verstanden, die die Selbstbestimmung der Patient*innen beeinträchtigen, nicht als Abwesenheit von als moralisch dringlich wahrgenommen Gründen oder Pflichten. Das Ergebnis könnte auch für ethische Überlegungen zur Frage Relevanz entfalten, ob und inwieweit Abstriche vom Ideal der klassischen (spezifischen) Form der informierten Einwilligung, z. B. im Falle der breiten Einwilligung oder gar einer systematischen Nutzung ohne explizite Einwilligung und nur mit Widerspruchsmöglichkeit (Opt-Out) ethisch akzeptabel sind. ${ }^{15}$ Es soll hier jedoch festgehalten werden, dass eine systematische Durchführung von SeConts sowohl mit als auch ohne Einwilligung stets in einen Governance Rahmen mit idealerweise systematischer Beteiligung und Vertretung von Patient*innen eingebettet sein sollte. Bezüglich der Relevanz für die rechtswissenschaftliche Diskussion handelt es sich bei den Elementen, welche die Zumutbarkeit bestimmen (Kosten für Patient*innen, Nutzen für Andere bzw. die Gesellschaft), auch um rechtlich entscheidende Kriterien für die zentrale Frage der Verhältnismäßigkeit bei der Forschungsnutzung klinischer Daten zur Durchführung von SeConts.

Danksagung Dieses Manuskript wurde im Rahmen des von der Deutschen Forschungsgemeinschaft (DFG) geförderten Projekts „Learning from Clinical Data (LinCDat)“ erstellt. Wir möchten uns bedanken bei unserem Projektpartner Prof. Dr. Kai Cornelius, LL.M. (Universität Heidelberg) und bei allen Mitgliedern der Sektion für Translationale Medizinethik (NCT Heidelberg) für fruchtbare Diskussionen, insbesondere bei Dr. phil. Christian Wendelborn für wertvolle Hinweise, sowie bei Vincent Lotz für Unterstützung bei der Recherche.

Förderung Gefördert durch die Deutsche Forschungsgemeinschaft (DFG) - 406103282.

Funding Open Access funding enabled and organized by Projekt DEAL.

Open Access Dieser Artikel wird unter der Creative Commons Namensnennung 4.0 International Lizenz veröffentlicht, welche die Nutzung, Vervielfältigung, Bearbeitung, Verbreitung und Wiedergabe in jeglichem Medium und Format erlaubt, sofern Sie den/die ursprünglichen Autor(en) und die Quelle ordnungsgemäß nennen, einen Link zur Creative Commons Lizenz beifügen und angeben, ob Änderungen vorgenommen wurden.

Die in diesem Artikel enthaltenen Bilder und sonstiges Drittmaterial unterliegen ebenfalls der genannten Creative Commons Lizenz, sofern sich aus der Abbildungslegende nichts anderes ergibt. Sofern das betreffende Material nicht unter der genannten Creative Commons Lizenz steht und die betreffende Handlung nicht nach gesetzlichen Vorschriften erlaubt ist, ist für die oben aufgeführten Weiterverwendungen des Materials die Einwilligung des jeweiligen Rechteinhabers einzuholen.

Weitere Details zur Lizenz entnehmen Sie bitte der Lizenzinformation auf http://creativecommons.org/ licenses/by/4.0/deed.de.

15 Zur Opt-out-Möglichkeit bei Datenverarbeitung auf gesetzlicher Grundlage ohne Einwilligung vgl. Spitz et al. (2021). 


\section{Einhaltung ethischer Richtlinien}

Interessenkonflikt M. Jungkunz, A. Köngeter, K. Mehlis, M. Spitz und C. Schickhardt geben an, dass kein Interessenkonflikt besteht. E.C. Winkler ist Mitglied der Schriftleitung der Zeitschrift Ethik in der Medizin.

Ethische Standards Für diesen Beitrag wurden von den Autor*innen keine Studien an Menschen oder Tieren durchgeführt. Für die aufgeführten Studien gelten die jeweils dort angegebenen ethischen Richtlinien.

\section{Literatur}

Arneson RJ (1982) The principle of fairness and free-rider problems. Ethics 92(4):616-633

Ballantyne A, Schaefer GO (2018) Consent and the ethical duty to participate in health data research. J Med Ethics 44(6):392-396. https://doi.org/10.1136/medethics-2017-104550

Bauhn P (2011) The extension and limits of the duty to rescue. Public Reason 3(1):39-49

Bayertz K (1998) Begriff und Problem der Solidarität. Suhrkamp, Frankfurt a.M.

Birnbacher D (2007) Analytische Einführung in die Ethik, 2. Aufl. de Gruyter, Berlin

Bohlken E (2011) Die Verantwortung der Eliten. Campus, Frankfurt a.M.

Brassington I (2007) John Harris' argument for a duty to research. Bioethics 21(3):160-168. https://doi. org/10.1111/j.1467-8519.2007.00539.x

Chadwick R, Berg K (2001) Solidarity and equity: new ethical frameworks for genetic databases. Nat Rev Genet 2(4):318-321. https://doi.org/10.1038/35066094

Cohen IG (2018) Is there a duty to share healthcare data? In: Cohen I, Lynch H, Vayena E, Gasser U (Hrsg) Big data, health law, and bioethics. Cambridge University Press, Cambridge, S 209-222 https://doi. org/10.1017/9781108147972.020

Cullity G (1995) Moral free riding. Philos Public Aff 24(1):3-34

Custers B, Dechesne F, Sears AM, Tani T, van der Hof S (2018) A comparison of data protection legislation and policies across the EU. Comput Law Secur Rev 34(2):234-243. https://doi.org/10.1016/j.clsr. 2017.09.001

Durkheim É (1992) Über soziale Arbeitsteilung - Studie über die Organisation höherer Gesellschaften. Suhrkamp, Frankfurt a. M.

Evans HM (2004) Should patients be allowed to veto their participation in clinical research? J Med Ethics 30(2):198-203. https://doi.org/10.1136/jme.2003.002444

Faden RR, Kass NE, Goodman SN, Pronovost P, Tunis S, Beauchamp TL (2013) An ethics framework for a learning health care system: a departure from traditional research ethics and clinical ethics. Hastings Cent Rep 43(1):S16-S27. https://doi.org/10.1002/hast.134

Fleischer H, Schickhardt C, Taupitz J, Winkler E (2016) Das Recht von Patienten und Probanden auf Herausgabe ihrer genetischen Rohdaten. MedR 34(7):481-491. https://doi.org/10.1007/s00350-0164319-9

Frankena WK (1972) Analytische Ethik. Eine Einführung. Deutscher Taschenbuch Verlag, München

Harris J (2005) Scientific research is a moral duty. J Med Ethics 31(4):242-248. https://doi.org/10.1136/ jme.2005.011973

Hoedemaekers R, Gordijn B, Pijnenburg M (2007) Solidarity and justice as guiding principles in genomic research. Bioethics 21(6):342-350. https://doi.org/10.1111/j.1467-8519.2007.00562.x

Hulsen T (2020) Sharing is caring - data sharing initiatives in healthcare. Int J Environ Res Public Health 17(9):3046. https://doi.org/10.3390/ijerph17093046

Jungkunz M (2018) Individualismus, komplexe Identität, Menschenrechte und Solidarität. Eine Re-Interpretation der Solidaritätstheorie Émile Durkheims wider den Atomismusvorwurf. Friedrich-Alexander-Universität Erlangen-Nürnberg, Erlangen (Dissertation)

Jungkunz M, Köngeter A, Mehlis K, Winkler EC, Schickhardt C (2021) Secondary use of clinical data in data-gathering, non-interventional research or learning activities: definition, types, and a framework for risk assessment. J Med Internet Res 23(6):e26631. https://doi.org/10.2196/26631

Jungkunz M, Köngeter A, Winkler EC, Mehlis K, Schickhardt C (2022) Sekundärnutzung klinischer Daten in datensammelnden, nicht-interventionellen Forschungs- und Lernaktivitäten - Begriff, Studientypen und ethische Herausforderungen. In: Richter G, Loh W, Buyx A, v Kielmansegg SG (Hrsg) Datenreiche Medizin und das Problem der Einwilligung. Ethische, rechtliche und sozialwissenschaft- 
liche Perspektiven. Springer, Berlin, Heidelberg, S 71-98 https://doi.org/10.1007/978-3-662-629871

Kant I (1785) Grundlegung zur Metaphysik der Sitten. Gesammelte Schriften, Bd. IV. Preussische Akademie der Wissenschaften, Berlin

Kant I (1797) Die Metaphysik der Sitten. Gesammelte Schriften, Bd. VI. Preussische Akademie der Wissenschaften, Berlin

v Kielmansegg SG (2020) Leitfrage 3: Welche rechtlichen Rahmenbedingungen braucht das Spenden von Daten? In: Strech D, v Kielmansegg SG, Zenker S, Krawczak M, Semler SC (Hrsg) Wissenschaftliches Gutachten „Datenspende“ - Bedarf für die Forschung, ethische Bewertung, rechtliche, informationstechnologische und organisatorische Rahmenbedingungen. Bundesministerium für Gesundheit, Berlin, S 85-124

Medizininformatik-Initiative (2017) MI-I-Kerndatensatz. https://www.medizininformatik-initiative.de/ sites/default/files/inline-files/MII_04_Kerndatensatz_1-0.pdf. Zugegriffen: 21. Juni 2021

Miller D (2020) The nature and limits of the duty of rescue. J Moral Philos 17(3):320-341. https://doi.org/ $10.1163 / 17455243-20193018$

Miller FG (2008) Research on medical records without informed consent. J Law Med Ethics 36(3):560-566. https://doi.org/10.1111/j.1748-720X.2008.304.X

Orentlicher D (2005) Making research a requirement of treatment. Why we should sometimes let doctors pressure patients to participate in research. Hastings Cent Rep 35(5):20-28. https://doi.org/10.1353/ hcr. 2005.0083

Ploug T (2020) In defence of informed consent for health record research-why arguments from 'easy rescue', 'no harm' and 'consent bias' fail. BMC Med Ethics 21(1):1-13. https://doi.org/10.1186/ s12910-020-00519-w

Porsdam Mann S, Savulescu J, Sahakian BJ (2016) Facilitating the ethical use of health data for the benefit of society: electronic health records, consent and the duty of easy rescue. Philos Trans A Math Phys Eng Sci. https://doi.org/10.1098/rsta.2016.0130

Prainsack B, Buyx A (2013) Solidarität - Der Versuch einer neuen Definition. In: Greif H, Weiss M (Hrsg) Ethics, Society, Politics. Proceedings of the 35th International Wittgenstein Symposium. De Gruyter, Berlin, S 575-596

Prainsack B, Buyx A (2017) Solidarity in biomedicine and beyond Bd. 33. Cambridge University Press, Cambridge

Rawls J (1979) Eine Theorie der Gerechtigkeit. Suhrkamp, Frankfurt a.M.

Rhodes R (2010) Rethinking research ethics. Am J Bioeth 10(10):19-36. https://doi.org/10.1080/ 15265161.2010 .519233

Rohland CJ (2021) Ein Weltrat für den Umweltschutz: Unser Planet ist noch zu retten! Springer, Wiesbaden

Ross WD (2002) The right and the good. Clarendon Press, Oxford

Saeltzer G (2004) Sind diese Daten personenbezogen oder nicht. Wie der Personenbezug von Daten, auch biometrischer, sich fundiert prüfen lässt. DuD 28(4):218-227

Schaefer GO, Emanuel EJ, Wertheimer A (2009) The obligation to participate in biomedical research. JAMA 302(1):67-72. https://doi.org/10.1001/jama.2009.931

Shapshay S, Pimple KD (2007) Participation in biomedical research is an imperfect moral duty: a response to John Harris. J Med Ethics 33(7):414-417. https://doi.org/10.1136/jme.2006.017384

Singer P (1972) Famine, affluence, and morality. Philos Public Aff 1(3):229-243

Spitz M, Cornelius K, Jungkunz M, Schickhardt C (2021) Rechtlicher Rahmen für eine privilegierte Nutzung klinischer Daten zu Forschungszwecken. MedR 39:499-504. https://doi.org/10.1007/s00350021-5898-7

Sturn R (2020) Private, öffentliche und meritorische Güter. In: Hiebaum C (Hrsg) Handbuch Gemeinwohl. Springer, Wiesbaden, S 1-13 https://doi.org/10.1007/978-3-658-21086-1_11-1

Taylor C (1994) Aneinander vorbei: Die Debatte zwischen Liberalismus und Kommunitarismus. In: Honneth A (Hrsg) Kommunitarismus. Campus, Frankfurt a. M., New York, S 103-130

Ter Meulen R (2017) Solidarity and justice in health and social care. Cambridge University Press, Cambridge

Yarborough M (2017) Why there is no obligation to participate in clinical research. J Law Med Ethics 45(3):327-332. https://doi.org/10.1177/1073110517737530 